product was found to sublime, when heated. The sublimed crystals melted at $260^{\circ}$ and were identified as anthraquinone.

Investigations on similar lines are being continued.

CALCUTTA, INDIA.

[Contribution from the Chemical, Laboratory of Presidency College.]

\title{
HALOGENATION. XVI. IODINATION BY MEANS OF NITRO- GEN IODIDE OR BY MEANS OF IODINE IN THE PRESENCE OF AMMONIA.
}

By Rasik lal datta and Nogendra Prosad.

Received December 26, 1916.

\section{Iodination of Phenols and Nitrophenols.}

The systematic iodination by means of nitrogen iodide, or by means of a potassium iodide solution of iodine in conjunction with liquor ammonia, which represents nitrogen iodide in the nascent state, was not attempted except in a few isolated cases. It has been found that not only the phenolic compound can be iodinated satisfactorily with the production of iodophenols but the nitrophenols can also be iodinated with the formation of iodonitrophenols. In the case of the nitrophenols, the further introduction of iodine atoms increases the acidic nature of the phenols and as the formation of iodonitrophenols takes place in the presence of ammonia, ammonium salts of varying degree of stability have been produced which have hitherto escaped isolation on account of the feebly acidic and inert nature of these iodonitrophenols. It is in the nascent state that the formation of the ammonium salt becomes possible, the direct union of ammonia with the iodonitrophenol being not successful.

The action of iodine and ammonia has been studied in the following cases: Phenol gives a quantitative yield of 2,4,6-triiodophenol. o-Cresol, $p$-cresol and $m$-cresol give in quantitative yield diiodo-o-cresol, diiodo- $p$ cresol and triiodo-m-cresol, respectively. From thymol, 6-iodo-thymol has been obtained. I,4,5-Xylenol has been found to yield a monoiododerivative. Phenolphthalein yields tetraiodophenolphthalein. o-Nitrophenol gives on iodination 2,4-diodo-6-nitrophenol which yields a stable ammonium salt. $m$-Nitrophenol yields 2 -iodo-3-nitrophenol which, however, yields an unstable ammonium salt which decomposes on keeping. From p-nitrophenol 2,6-diiodo-4-nitrophenol has been obtained which does not give any ammonium salt. 3-Nitro-I,4-cresol has been found to yield 5-iodo-3-nitro-I,4-cresol and a stable ammonium salt. The special feature of this reagent is that in all cases quantitative yields of the products are obtained. In view of the fact that both ammonia and iodine in the mother liquor could be conveniently recovered, these methods would serve as the best modes of preparing these iodophenols and iodonitrophenols on the large scale. 
Iodination of Phenol. Preparation of Triiodophenol.-Triiodophenol has been prepared by the action of iodine and caustic alkali on salicylic acid $^{1}$ as also by the action of iodine and iodic acid on same. ${ }^{2}$ Schützenberger $^{3}$ obtained it by the interaction of phenol and iodine chloride. Baeyer \& Co. ${ }^{4}$ prepared it by the action of caustic soda on diodophenol iodide. Schall ${ }^{5}$ showed that on adding iodine to sodium phenate suspended in carbon bisulfide solution, a mixture of mono-, di- and triiodophenol is formed which can be separated only with difficulty. Richard ${ }^{6}$ obtained a mixture of mono-, di- and triiodophenols by the action of an aqueous solution of the phenol containing either sodium bicarbonate or sodium hydrogen phosphate or sodium acetate. Messinger ${ }^{7}$ prepared it by the action of hot potash on phenol and iodine. Walkie ${ }^{8}$ gives a method for the volumetric estimation of phenol based upon the formation of triiodophenol in the presence of sodium carbonate. Lepetit ${ }^{3}$ prepared it by the action of nitrogen iodide on an alkaline solution of phenol.

Now triiodophenol is formed almost quantitatively by the action of an iodine solution on an ammoniacal solution of phenol. It has been found that the use of solid nitrogen iodide by Lepetit is not very convenient for dealing with reasonably large quantities of phenol and the triiodophenol produced by his method is almost invariably contaminated with some secondary oxidation products of phenol from which it is quite difficult to get a pure product. The preparation of triiodophenol is carried out as follows: Phenol is dissolved in an excess of strong aqueous ammonia and to it a strong solntion of iodine in potassium iodide is gradually added when a white flocculent precipitate deposits at the bottom of the vessel. The addition of iodine is continued until the color of iodine persists and this is the case when the reaction becomes complete. The precipitate is next filtered at the pump, dried and crystallized from glacial acetic acid in which it is moderately soluble. White feathery crystals of $2,4,6$-triiodophenol are obtained melting at $156^{\circ}$. The reaction is quite quantitative as has been found by a volumetric estimation with starch as indicator. In a quantitative experiment it has been found that 0.2663 g. required for complete precipitation $2.16 \mathrm{~g}$. iodine, while 2.16 of iodine is theoretically equivalent to $0.2665 \mathrm{~g}$. phenol.

o-Cresol. Preparation of Diiodo-o-cresol.-Diiodo-o-cresol was pre-

${ }^{1}$ Lautemann, $A n n$., I20, 307 (1861).

${ }^{2}$ Kekule, Ibid., I3I, 23 I (1864).

3 Schützenberger, Jahrb., 1865, 524.

D. R. P. 52,828 .

EBer., I6, 1897 (1883).

${ }^{6} J$. Pharm. Chem., [vi] 15, 217 (1902).

7 Ber, 22, 2312 (1889).

${ }^{8}$ J. Soc. Chem. Ind., 30, 398 (r91r).

- Gace, 20, 105 (1890). 
viously obtained by the action of solid iodine on an ammoniacal solution of 0 -cresol. ${ }^{1}$ It has also been obtained by Pence ${ }^{2}$ by the action of iodine on an alkaline solution of $o$-cresol containing sodium iodate. It has been prepared more conveniently thus. $o$-Cresol is dissolved in a moderately large excess of ammonia and to it a solution of iodine in potassium iodide is gradually added with shaking when a white flocculent precipitate is formed which increases as the addition is continued. When no more iodine is taken up, the precipitate is filtered, washed, dried and recrystallized from glacial acetic acid. White needle-shaped crystals of diiodo$o$-cresol are obtained melting at $67^{\circ} .4 .4 \mathrm{~g}$. of cresol gave I $2.4 \mathrm{~g}$. of diiodo-o-cresol.

$m$-Cresol. Preparation of Triiodo- $m$-cresol.-A mixture of monoiodoand diiodo- $m$-cresol has been obtained by Willgerodt and Kornblum ${ }^{3}$ by the addition of solid iodine to a solution of $m$-cresol in ammonia. Pence ${ }^{2}$ also noticed while describing some volumetric methods for the estimation of $o$ - and $p$-cresols that by the action of iodine and alkali on $m$-cresol, a definite iodo compound could not be obtained. Kalle \& Co. ${ }^{4}$ prepared triiodo-m-cresol by the action of 3 mols caustic soda and 6 mols iodine on I mol of cresol. Triiodo- $m$-cresol is obtained by Bayer $\& \mathrm{Co}^{5}$ by the action of iodine in potassium iodide on sodium derivative of $m$-cresol. This has been produced quite readily and in a pure state by the action of iodine solution on an ammoniacal solution of $m$-cresol. Cresol is dissolved in an excess of moderately strong ammonia and to it iodine solution is gradually added which is taken up very readily-much more readily than in the case of either the $o$ - or the $p$-compound. After it has taken up a good quantity of iodine, a white precipitate begins to deposit which gradually becomes very bulky. As soon as the color of iodine persists, the reaction is stopped. The precipitate is filtered, washed and dried. On crystallization from glacial acetic acid, triiodo- $m$-cresol is obtained in small white needle-shaped crystals melting at $122^{\circ}$. The yield obtained is quantitative.

$p$-Cresol. Preparation of 3,5-Diiodo-p-cresol.-By the addition of iodine to the sodium derivative of $p$-cresol, Schall and Drulle ${ }^{6}$ obtained a mixture of 3 -iodo- $p$-cresol and 3,5-diiodo-p-cresol. The diiodo- $p$-cresol is also formed by the action of iodine on an alkaline solution of $p$-cresol containing sodium acetate. ${ }^{7}$ This has been prepared by the action of iodine in ammoniacal solution of $p$-cresol, as follows: $p$-Cresol is

1 Willgerodt and Kornblum, J. prakt. Chem., [2] 39, 295 (1890).

2 Pence, J. Ind. Eng. Chem., 4, 518 (I912).

3 J. prakt. Chem., [2] 39, 297.

- D. R. P. 106,504.

S Ibid., 72,996.

${ }^{6}$ Ber., I7, 2534 (1884).

${ }^{7}$ Pence, Loc. cit. 
taken in a flask and to it a large excess of ammonia is added when it is dissolved. A concentrated solution of iodine is very gradually added with vigorous shaking. At first it is completely taken up, but gradually a white flocculent precipitate begins to deposit. When no more iodine is taken up, the operation is stopped. The precipitate is filtered, washed, dried and crystallized from glacial acetic acid in which it is very soluble and does not crystallize out readily. On leaving the solution to evaporate slowly in the open air, 3,5-diodo-p-cresol is obtained in needles melting at $62^{\circ}$. Io $\mathrm{g}$. of $p$-cresol gave $30 \mathrm{~g}$. of the iodo derivative.

Thymol. Preparation of 6-Iodothymol.-6-Iodothymol was obtained by Willgerodt and Kornblum ${ }^{1}$ by the action of solid iodine to a solution of thymol in ammonia mixed with alcohol. Messinger and Vortman ${ }^{2}$ obtained diiodothymol by the action of iodine on an alkaline solution of Willgerodt's iodothymol. 6-Iodothymol has been prepared more readily by the action of a potassium iodine solution of iodine to an ammoniacal solution of thymol. To an aqueous solution of thymol containing a little caustic soda, strong aqueous ammonia is added and the mixture is warmed on the water bath. To this iodine solution is gradually added in small quantities at a time and allowing time for each batch of iodine to be taken up. The reaction is to be carried out in a warm solution or a precipitate of nitrogen iodide is formed. The reaction is stopped when the liquid is permanently brown. The precipitate is filtered, washed and dried. On recrystallization from alcohol, 6-iodothymol is obtained in crystals melting at $69^{\circ}$.

Xylenol $\left(\mathrm{CH}_{3}: \mathrm{CH}_{3}: \mathrm{OH}=\mathrm{I}: 4: 5\right)$. Preparation of Monoiodoxylenol.--This substance had not been previously iodinated. The xylenols are not so readily iodinated as the lower members. Xylenol is dissolved in a very large excess of ammonia. The solution is then heated on the water bath and to it iodine solution is added which is taken up and at the same time a dirty white precipitate begins to be deposited. The addition of iodine is continued with warming on the water bath until no more of it is taken up. At the end a whitish yellow powder is left behind which is not soluble in the ordinary solvents but dissolves in benzene and toluene from which it does not crystallize well but is deposited as a crust. It does not give any melting point, but decomposes on heating and ultimately chars. The compound on analysis proved to be a monoiodo derivative of $1: 4: 5$-xylenol, the determination of the constitution of which is deferred for the present.

0.1933 gave $0.2713 \mathrm{CO}_{2}$ and $0.0992 \mathrm{H}_{2} \mathrm{O} ; \mathrm{C}, 38.62 ; \mathrm{H}, 5.70$.

0.1828 gave 0.1733 AgI; I, 51.23 .

Calc. for $\mathrm{C}_{6} \mathrm{H}_{2} \mathrm{I}\left(\mathrm{CH}_{3}\right)_{2} \mathrm{OH}: \mathrm{C}, 38.7 \mathrm{I} ; \mathrm{H}, 3.73 ; \mathrm{I}, 5 \mathrm{I} .2 \mathrm{I}$.

1 J. prakt. Chem., [2] 39, 290.

\& Ber, 22, 2312 (1889). 
o-Nitrophenol. Preparation of 2,4-Diiodo-6-nitrophenol and Its Ammonium Salt. - This substance is iodinated by Körner ${ }^{1}$ by means of iodine and iodic acid in alkaline solution. When the iodination of 0 -nitrophenol is carried out by means of an iodine solution in the presence of aqueous ammonia, the ammonium salt is formed from which, by crystallization from glacial acetic acid, 2,4-diiodo-6-nitrophenol is obtained. A preliminary experiment indicated that the iodination could not be carried out in a cold solution, as in that case nitrogen iodide is precipitated which is not taken up in the cold. Some nitrophenol is dissolved in an excess of aqueous ammonia with warming on the water bath. Then potassium iodide solution of iodine is gradually added. The color of the solution changes from orange-red to dark brown. After some time an orangered precipitate begins to deposit and increases in amount as the action is continued. During the course of the reaction, the mixture is heated on the water bath from time to time. The addition of iodine is stopped when no more precipitates are formed and the solution assumes a permanently dark color. The orange-red precipitate thus obtained is filtered, washed and dried. It is somewhat soluble in hot water from which it crystallizes in small orange-red needles. It consists of the ammonium salt of 2,4-diiodo-6-nitrophenol melting at $210^{\circ}$ with decomposition.

0.1008 gave $5.8 \mathrm{cc}$. $\mathrm{N}_{2}$ at 22 and $760 \mathrm{~mm}$.: $\mathrm{N}, 6.49$.

Calc. for $\mathrm{C}_{6} \mathrm{H}_{2} \mathrm{I}_{2}\left(\mathrm{NO}_{2}\right)\left(\mathrm{ONH}_{4}\right): \mathrm{N}, 6.86$.

A portion of the crude ammonium salt is recrystallized from glacial acetic acid, when 2,4-diiodo-6-nitrophenol is obtained in deep yellow needle-shaped crystals melting at $98^{\circ}$.

$m$-Nitrophenol. Preparation of 2-Iodo-3-nitrophenol and Its Ammonium Salt.-Schlieper ${ }^{2}$ obtained 2-iodo-3-nitrophenol by the action of iodine and mercuric oxide on $m$-nitrophenol. On addition of $m$-nitrophenol with iodine and ammonia, an unstable ammonium salt of 2-iodo-3-nitrophenol was initially formed from which 2-iodo-3-nitrophenol could be readily obtained. The reaction is carried out by dissolving $m$-nitrophenol in a hot solution of ammonia and by adding the iodine solution gradually. At the end a reddish brown precipitate is formed. The precipitate is left to stand overnight to allow it to settle completely. The precipitate; on being washed and dried, is found to melt at $165-170^{\circ}$ and consists of the ammonium salt. It cannot, however, be crystallized from water, as it is decomposed by water and is not soluble in it. The nitrogen as estimated immediately after preparation corresponds to the pure ammonium salt.

Found: N, 9.23. Calc., N, 9.93.

${ }^{1}$ Jahrb., 1867, 617.

${ }^{2}$ Ber., 26, 2467 (1893). 
If the salt is allowed to stand, it gradually decomposes with loss of ammonia. A nitrogen estimation made after allowing the salt to stand for some days gave a lower value of nitrogen.

\section{Found: N, 6.02. Calc., N, 9.93.}

The crude ammonium salt on recrystallization from glacial acetic acid gave fine yellow needles of 2-iodo-3-nitrophenol melting at $134^{\circ}$.

$p$-Nitrophenol. Preparation of 2,6-Diiodo-4-nitrophenol. -This is iodinated by Körner ${ }^{1}$ by means of iodine and iodic acid in alkaline solution with the formation of 2,6-diiodo-4-nitrophenol. It is also obtained by the action of iodine and mercuric oxide on the $o$-sulfonic derivative of $p$-nitrophenol. ${ }^{2} p$-Nitrophenol appears to be less reactive than $o$-nitrophenol. Some $p$-nitrophenol is dissolved in an excess of moderately strong aqueous ammonia by warming on the water bath. Then iodine solution in potassium iodide is gradually added and at first it seems to be readily taken up but gradually the color of the solution becomes very dark. After continued addition with shaking from time to time, the solution slowly deposits red powdery crystals. It is heated on the water bath for some time, allowed to stand overnight, filtered, washed and dried. Unlike the other iodonitrophenols, it does not yield the pure ammonium salt, but in this case it is obtained in a quite decomposed state whose identity could not be established. On crystallization from glacial acetic acid, light yellow powdery crystals of 2,6-diiodo-4-nitrophenol, melting at $157^{\circ}$, are obtained which gradually turn red on exposure to air.

3-Nitro-1,4-cresol. Preparation of 5-Iodo-3-nitro-1,4-cresol and Its Ammonium Salt.-This substance has not been iodinated before. On iodination with iodine in the presence of ammonia it yields the ammonium salt of 5-iodo-3-nitro-I,4-cresol, which yields the iodo compound by crystallization from glacial acetic acid. 3-Nitro- 1,4 -cresol is first dissolved in an excess of strong ammonia warming on the water bath, the solution having an orange-red color due to the formation of the ammonium salt of 3-nitro- $\mathrm{r}, 4$-cresol. Then a solution of iodine in potassium iodide is gradually added little by little with vigorous shaking. A precipitate begins to be thrown down at once, and the operation is stopped when no more iodine is taken up. The flask is heated on the water bath when the precipitate formed dissolves completely. On allowing it to cool, the contents of the flask solidify to small needle-shaped orange-red crystals. These are filtered and washed with only a very small quantity of water since they appear to be soluble in this solvent. These were then recrystallized from hot water, giving m. p. $195^{-200^{\circ}}$. Analysis indicates that it is the ammonium salt of monoiodo-3-nitro-r,4-cresol.

\footnotetext{
1Z. Chem, I868, 324 .

Post, Ann., 205, 91 (1880).
} 
0.2105 gave $0.2182 \mathrm{CO}_{2}$ and $0.0991 \mathrm{H}_{2} \mathrm{O} ; \mathrm{C}, 28.27 ; \mathrm{H}, 5.28$.

0.0780 gave $6.6 \mathrm{cc} . \mathrm{N}_{2}$ at $30^{\circ}$ and $760 \mathrm{~mm}$.; N, 9.74.

0.147 I gave 0.1 I $58 \mathrm{AgI} ; \mathrm{I}, 42.56$.

Calc. for $\mathrm{C}_{6} \mathrm{H}_{2} \mathrm{I}\left(\mathrm{CH}_{3}\right)\left(\mathrm{NO}_{2}\right)\left(\mathrm{ONH}_{4}\right): \mathrm{C}, 28.38 ; \mathrm{H}, 3.04 ; \mathrm{N}, 9.46 ; \mathrm{I}, 42.90$.

A part of the substance was recrystallized from glacial acetic acid. Yellow needlè-shaped crystals were obtained melting at $83.5^{\circ}$, whose composition corresponds to the monoiodo compound. Since one orthoand para-positions are already occupied, the substance is 5 -iodo-3-nitroI,4-cresol.

0.1573 gave $0.1735 \mathrm{CO}_{2}$ and $0.0313 \mathrm{H}_{2} \mathrm{O} ; \mathrm{C}, 30.08: \mathrm{H}, 2.2 \mathrm{I}$.

0.2316 gave $10.2 \mathrm{cc} . \mathrm{N}_{2}$ at $30^{\circ} \mathrm{C}$; $\mathrm{N}, 5.07$.

0.1439 gave $0.1208 \mathrm{AgI} ; \mathrm{I}, 45.36$.

Calc. for $\mathrm{C}_{6} \mathrm{H}_{2} \mathrm{I}\left(\mathrm{CH}_{3}\right)\left(\mathrm{NO}_{2}\right)(\mathrm{OH}): \mathrm{C}, 30 . \mathrm{II} ; \mathrm{H}, 2.15: \mathrm{N}, 5.01 ; \mathrm{I}, 45.52$.

Phenolphthalein. Preparation of Tetraiodophenolphthalein.-Phenolphthalein has been previously iodinated by the action of iodine to an alkaline solution of phenolphthalein ${ }^{1}$ with the formation of tetraiodophenolphthalein. This is prepared more readily and in better yield by the action of iodine on an ammoniacal solution of phenolphthalein. Some phenolphthalein is taken in a flask and moderately dilute ammonia is added, when most of it dissolves on shaking. The last traces are dissolved by heating on the water bath. To the pink solution a solution of iodine in potassium iodide is gradually added while the solution is still warm. The iodine seems to be taken up at first but when the solution has somewhat cooled, nitrogen iodide is precipitated. After continued warming on the water bath, the nitrogen iodide disappears and a light yellow powdery precipitate begins to form and settle at the bottom. When no more iodine is taken up, which is known as usual by the color of the iodine solution, the precipitate is filtered, washed and dried. The substance, on examination, proved to be tetraiodophenolphthalein obtained by Classen and Lob. This was confirmed by an halogen estimation:

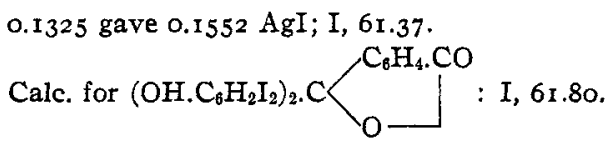

Iodination of the Phenolic Acids.

The phenolic acids could be less readily iodinated by nitrogen iodide than the phenols and nitrophenols. In these cases besides the formation of monoiodo- or diiodo derivatives, secondary products are always formed of a complex character due to a peculiar condensation of the iodophenolic acids.

Salicylic Acid. Preparation of 5-Iodosalicylic Acid.-Salicylic acid had been previously iodinated by Lauteman ${ }^{2}$ by the action of iodine in

${ }^{1}$ Classen and Lob, Ber., 28, 1605 (1895).

2 Ann., 120, 302 (1861). 
the presence of alkali with the formation of 5 -iodosalicylic acid and 3,5diiodosalicylic acid. By the action of iodine in the presence of ammonia, the 5 -iodosalicylic acid is the main product, accompanied by a complex condensation product. Salicylic acid is dissolved in a large volume of ammonia with shaking. Iodine solution is then gradually added, when it is found that the iodine is taken up readily. It should be shaken thoroughly to avoid the collection of solid nitrogen iodide. After some time a thick white precipitate begins to collect which increases in amount as the reaction is completed. When no more iodine is taken up, the mixture is allowed to stand for some time at the ordinary temperature, and finally, the mixture is warmed on the water bath. The precipitate is next washed thoroughly with water to remove the soluble iodides. It is recrystallized from glacial acetic acid when an insoluble pinkish product was obtained. From glacial acetic acid solution, white crystals were obtained, which, after two or three recrystallizations, gave a product melting at $193^{\circ}$, which was identified to be 5-iodo-salicylic acid. The pinkish product is under investigation.

m-Hydroxybenzoic Acid. Preparation of 6-Iodo-3-hydroxybenzoic Acid.- $m$-Hydroxybenzoic acid has not been previously iodinated by means of iodine. By the action of iodine in the presence of ammonia, 6-iodo-3-hydroxybenzoic acid has been obtained. This compound has been previously prepared from 6-amido-3-hydroxybenzoic acid by means of Sandermeyer's reaction.'

$m$-Oxybenzoic acid is dissolved in a large excess of ammonia and to it iodine solution is gradually added with shaking. On the addition of the first few drops of iodine, a black precipitate of nitrogen iodide is deposited at first but when the flask is shaken very thoroughly it gradually disappears. The rate of the solution of the precipitated nitrogen iodide in this case is far less than in the case of salicylic acid. More of iodine solution is then added, but only a small quantity at a time, so that a large quantity of nitrogen iodide may not accumulate. As more and more iodine solution is added with shaking, the reaction takes place more readily, but after a time, when it has taken up a considerable quantity of iodine, the reaction slackens again. When the reaction is complete, it is found that a small amount of a viscous precipitate is formed. This is filtered off, and the mother liquor is acidified with hydrochloric acid, when a flocculent precipitate is obtained. This, on recrystallization from glacial acetic acid, is found to be 6 -iodo- 3 -hydroxybenzoic acid melting at $196^{\circ}$. It appears that this compound is soluble in ammonia with the formation of a soluble ammonium salt from which the substance is precipitated by acidifying with hydrochloric acid.

Timpricht, $A n_{n}, 263,234(18,1)$. 
p-Hydroxybenzoic Acid. Preparation of 3,5-Diiodo-6-hydroxybenzoic Acid.-p-Hydroxybenzoic acid gives, on iodination, a good yield of 3,5-diiodo-p-hydroxybenzoic acid. This has been previously obtained by Peltzer ${ }^{1}$ by the action of iodine and iodic acid on $p$-hydroxybenzoic acid. To a solution of $p$-hydroxybenzoic acid in ammonia, iodine solution is gradually added when at first a black precipitate of nitrogen iodide is obtained, which disappears slowly on shaking. The rate of disappearance of nitrogen iodide in this case is much slower than in the other two cases. The reaction is facilitated by warming on the water bath from time to time. At the end, it is found that some precipitate has collected. On acidifying the solution with dilute hydrochloric acid, a further quantity of precipitate is formed. The precipitates are filtered and recrystallized from glacial acetic acid when needle-shaped crystals of 3,5-diodo-6hydroxybenzoic acid are obtained melting at $140^{\circ}$.

If the reaction be continued further with fresh quantities of iodine, a large amount of an insoluble product is formed which is under investigation.

p-Hydroxyphenylarsenic Acid. Preparation of Its Monoiodo Derivative.-This substance has not been iodinated before. To a hot solution of phenylarsenic acid in ammonia, iodine solution is gradually added. The addition of iodine is continued until there is no more decolorization of same. As the reaction proceeds, small needle-shaped crystals begin to deposit. On allowing the mixture to cool a further amount of copious crystalline precipitate collects. The precipitate is then filtered and recrystallized from glacial acetic acid in needle-shaped crystals melting at $158^{\circ}$. Analysis indicates it to be a monoiodo derivative of $p$-hydroxyphenylarsenic acid.

$$
\begin{aligned}
& \text { 0.163 I gave } 0.1249 \mathrm{CO}_{2} \text { and } 0.05 \mathrm{I}_{4} \mathrm{H}_{2} \mathrm{O} ; \mathrm{C}, 20.80 ; \mathrm{H}, 3.50 \text {. } \\
& 0.2018 \text { gave } 0.1415 \mathrm{AgI} ; \mathrm{I}, 37.87 . \\
& \text { Calc. for } \mathrm{C}_{6} \mathrm{H}_{8} \mathrm{I}(\mathrm{OH}) \mathrm{AsO}(\mathrm{OH})_{2}: \mathrm{C}, 20.92 ; \mathrm{H}, 1.74 ; \mathrm{I}, 36.92 \text {. }
\end{aligned}
$$

It has been found that when the moist precipitate is boiled with water for a considerable length of time, it is transformed into a pink-colored substance melting at $230-240^{\circ}$ which is under investigation.

\section{General Iodinations.}

Dimethylpyrone. Preparation of 2,6-Diiodolutidone and Its Hydrochloride.-Dimethylpyrone was not previously iodinated. On treating dimethylpyrone with iodine solution in the presence of ammonia, two products are mainly formed, viz., iodoform and diiodolutidone. Iodoform is formed due to rupture of the dimethylpyrone ring in the presence of nitrogen iodide. Diiodolutidone is obtained as the main product. During the course of the reaction it was also observed that a shining mica-like crystalline substance is formed as an intermediate product which

${ }^{1}$ Ann., i46, 294 (1868). 
however, is ultimately converted into diiodolutidone. The formation of diiodolutidone may be represented thus:

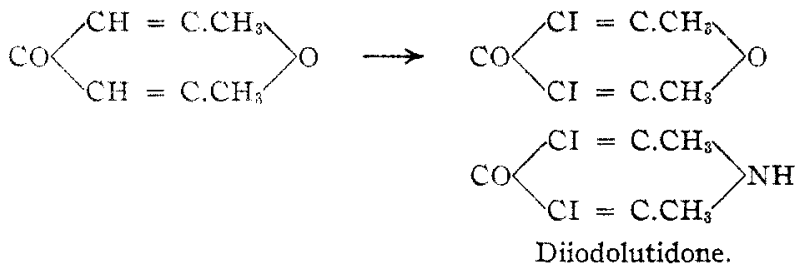

As a basic stubstance it forms an hydrochloride which has been isolated as a crystalline substance.

$5 \mathrm{~g}$. of dimethylpyrone are dissolved in $50 \mathrm{cc}$. of water and the solution is warmed on the water bath. When the temperature reaches about $70^{\circ}$, the solution is taken from the water bath and about $15 \mathrm{cc}$. of strong aqueous ammonia are added and then iodine solution. Nitrogen iodide is precipitated at first, but it disappears on shaking, giving rise to a yellowish solution. More iodine is added which is now taken up more readily and when any brown coloration is formed, the flask is warmed on the water bath with shaking, when it disappears. After some time, when the flask becomes a little cooler, shining mica-like white crystals are observed to appear, but these again disappear partially on heating, leaving a yellow powder behind. In this way, the addition of iodine is continued till the white solid increases no more and the operation is stopped, when there is a permanent brown color. A strong odor of iodoform is also noticed and crystals of iodoform are deposited on the sides of the flask. The precipitate is filtered, washed repeatedly with water and extracted thrice with hot ammonia. Next it is repeatedly extracted with alcohol to free it completely from iodoform. It decomposes at $230-235^{\circ}$. It is not soluble in any of the ordinary solvents, but is decomposed by nitric and sulfuric acids.

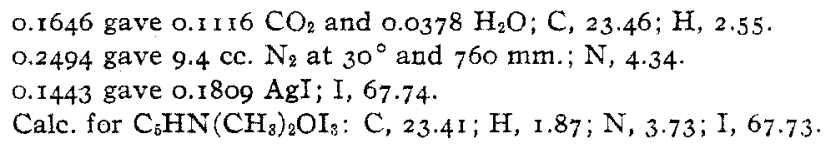

Hydrochloride.-The product was boiled with concentrated hydrochloric acid in a flask when it dissolved. On cooling, light yellow needleshaped crystals were obtained. It was filtered and dried by means of a porous pot. It was not washed with water since it is hydrolyzed by it. It does not melt.

0.1225 gave $0.0897 \mathrm{CO}_{2}$ and $0.0254 \mathrm{H}_{2} \mathrm{O} ; \mathrm{C}, 19.96 ; \mathrm{H}, 2.30$.

$0.256 \mathrm{I}$ gave $7.8 \mathrm{cc} \mathrm{N}_{2}$ at $30^{\circ}$ and $760 \mathrm{~mm}$. : N, 3.50.

$0.2 \mathrm{IO} 2$ gave $0.3105 \mathrm{AgCl}+\mathrm{AgI}$ and $0.2172 \mathrm{AgCl} ; \mathrm{Cl}, 8.4 \mathrm{I} ; \mathrm{I}, 61.3 \mathrm{I}$.

Calc. for $\mathrm{C}_{3} \mathrm{HNN}\left(\mathrm{CH}_{3}\right)_{2} \mathrm{OI}_{2} . \mathrm{HCl}: \mathrm{C}, 20.44 ; \mathrm{H}, 1.94 ; \mathrm{N}, 3.41 ; \mathrm{Cl}, 8.64 ; \mathrm{I}, 61.8$. 
Pyrrol. Preparation of Tetraiodopyrrol and the Estimation of Pyrrol. - By the iodination of pyrrol in the presence of caustic potash tetraiodopyrrol has been obtained by Ciamician and Silber. ${ }^{1}$ The same compound is also produced by the action of iodine on potassium pyrrol. ${ }^{2}$

It has been found that tetraiodopyrrol is produced in quantitative yield by the action of iodine on pyrrol dissolved in ammonia. It has been found that the reaction could be used as a method for the volumetric estimation of pyrrol. For the preparation of tetraiodopyrrol, $5 \mathrm{cc}$. of pyrrol are shaken up with about $200 \mathrm{cc}$. of water and then ammonia is added in sufficient quantity. To this the iodine solution is gradually added when a temporary precipitate is formed which dissolves on shaking. As the addition of iodine solution is continued, a voluminous white precipitate is obtained which appears to be little dirty. When no more iodine is taken up, the precipitate is filtered, washed and crystallized from glacial acetic acid below $100^{\circ}$, as boiling acetic acid partly decomposes the substance. It is obtained in beautiful yellowish crystals melting with decomposition between $140-10^{\circ}$.

Found: I, 88.35. Calc.: I, 88.97.

For the volumetric estimation of pyrrol, the following details may be carried out: To an ammoniacal solution of pyrrol a standard solution of iodine is added when there is quantitative precipitation of all the pyrrol as tetraiodopyrrol. The slightest excess of iodine is ascertained by starch solution as outside indicator. The accuracy of the method depends upon the high dilution of the iodine solution; $0.5 \mathrm{~N}$ is a suitable solution to use. The reaction that takes place is represented thus:

$$
\mathrm{C}_{4} \mathrm{H}_{4} \mathrm{NH}+{ }_{4} \mathrm{I}_{2}+{ }_{4} \mathrm{NH}_{3}=\mathrm{C}_{4} \mathrm{I}_{4} \mathrm{NH}+{ }_{4} \mathrm{NH}_{4} \mathrm{I}
$$

Each pyrrol molecule is equivalent to eight atoms of iodine.

The results of several determinations have been found to be 99.67 , $99.56,99.81,99.76 \%$, respectively, in pure samples of pyrrol.

Acetylene. Preparation of Tetraiodoethylene.-By the action of acetylene on nitrogen iodide under water, a quantitative yield of tetraiodoethylene has been obtained. This ethylene derivative has not hitherto been prepared directly from acetylene by the action of iodine in the presence of other reagents, diiodoacetylene being generally produced. Nitrogen iodide is first obtained as a black precipitate by adding strong ammonia to a potassium iodide solution of iodine. It is washed thoroughly with water by decantation, is placed under water and acetylene is slewly passed with shaking from time to time. At first, the greenish yellow supernatant water begins to decolorize. On shaking the color reappears, due to the dissolution of some nitrogen iodide, which is again decolorized in

1 Ciamician and Silber, Ber., 18, I 766 (1885).

2 Ciamician and Denstedt, Ibid., 15, 2582 (1882). 
a similar way. The interaction becomes more rapid as the reaction proceeds. Crystals begin to appear which gradually increase until the whole of the nitrogen iodide is converted into a mass of dirty white, fine crystals, which on the further action of the gas turn orange-yellow. The substance is recrystallized from ether when small light yellow needle-shaped crystals of tetraiodoethylene melting at $185^{\circ}$ are obtained. The yield is quantitative; the nitrogen iodide is completely transformed into the iodo derivative.

Phenylacetylene. Preparation of Triiodostyrol.-By the action of iodine in potassium iodide, diiodostyrol could be obtained. Triiodostyrol has not hitherto been prepared directly from phenylacetylene, which, however, has been prepared by Liebermann and Sachse ${ }^{1}$ by the action of iodine solution in potassium iodide on phenylacetylene silver. The hydrogen of phenylacetylene is incapable of substitution directly by iodine which has been found possible with nitrogen iodide. Phenylacetylene is warmed on the water bath in a flask and to it moist nitrogen iodide, which can, however, be handled freely, is added in small quantities at a time when the nitrogen iodide is taken up readily. The addition of nitrogen iodide is continued until no more of it is taken up. On cooling, the contents of the flask solidify completely. This is sucked to free it from mother liquor, and crystallized from glacial acetic acid. Light yellow needle-shaped crystals of triiodostyrol, melting at $108^{\circ}$, are obtained.

\section{Nitrogen Iodide as an Oxidizing Agent.}

Nitrogen iodide is a stronger oxidizing agent than iodine. Its oxidizing action has been chiefly observed in the case of the phenols, some aldehydes and a few other substances. Two cases have been found to give definite results at present. Hydroquinone is oxidized to quinhydrone and benzaldehyde to benzoic acid. Many other results have been obtained, which being not very definite, have been left out for the present.

Hydroquinone. Preparation of Quinhydrone.-Hydroquinone is completely oxidized to quinhydrone. Since ammonia has a direct action on hydroquinone, coloring it brown, the action has been tried with solid nitrogen iodide carefully freed from all ammonia by washing. To a strong solution of hydroquinone in a test tube, nitrogen iodide is added and the solution is shaken, when dark green, crystalline precipitates are observed to form at once and the whole solution becomes clogged with them in a short time. These are separated, washed and crystallized when pure crystals of quinhydrone melting at $\mathrm{I}_{7} \mathrm{I}^{\circ}$ are obtained.

Benzaldehyde. Formation of Benzoic Acid.-Benzaldehyde is completely oxidized to benzoic acid. To some benzaldehyde pure nitrogen iodide is gradually added when a vigorous reaction takes place with the

$$
\text { : Ber, 24, +1 15 (1801) }
$$


complete decomposition of nitrogen iodide and evolution of much heat. Care should be taken to add nitrogen iodide in small quantities at a time for fear of explosion. ${ }^{1}$ At the end of the reaction, the liquid is diluted with a little water and a copious amount of a white crystalline solid is obtained. This is separated and sublimed when crystals of benzoic acid, melting at $120^{\circ}$, have been obtained.

\section{The Formation of Iodoform.}

Chattaway ${ }^{2}$ obtained iodoform from acetone and acetophenone by the action of nitrogen iodide. It has been found that iodoform is formed as a product of systematic action of nitrogen iodide on the ketones. But if the reaction be carried out with concentrated solutions and at lower temperatures, iodoketones are formed, the successful isolation of which has been abandoned for the present on account of very great difficulties. The following cases of the formation of iodoform through nitrogen iodide have been studied:

Acetone.-When solid nitrogen iodide or liquor ammonia and iodine are added to acetone, the iodine is taken up very readily with the formation of some iodoacetone which has a powerful odor attacking the eyes, causing an abundance of tears. This reaction takes place only when a very small quantity of ammonia is present. The preparation of iodoform has been described previously by Chattaway. ${ }^{3}$

Methylethylketone.-By the action of iodine and ammonia in strong solution of the ketone, iodoketone is obtained which attacks the eyes powerfully, the isolation of which, as indicated above, is deferred for the present. $2 \mathrm{cc}$. of the ketone are diluted with $50 \mathrm{cc}$. of water and to this Io cc. of liquor ammonia are added. On the addition of a strong solution of iodine in potassium iodide, nitrogen iodide is precipitated as a black mass. This is then warmed on the water bath, when the precipitated nitrogen iodide disappears on shaking. When the reaction has proceeded thus far, no nitrogen iodide is precipitated but the iodine is at once taken up. After some time the liquid becomes yellow and deposits a yellow crystalline precipitate. This is iodoform and by crystallization from alcohol it is obtained quite pure, melting at $\mathrm{I}_{1} 9^{\circ}$, yield being $\mathrm{I} .5 \mathrm{~g}$.

Diethylketone.-Like the other ketones, diethylketone when treated with nitrogen iodide, yields the iodoketones, having very irritable odor. On effecting the interaction in a dilute solution, iodoform is formed in good quantity. It has been observed that as we rise higher and higher in the homologous series of the ketones, the tendency of the formation of iodoform gradually diminishes and the yields also gradually become less. The following method has been employed for the preparation of

1 This however has never been found to take place.

2 J. Chem. Soc., 103, 1986 (1913).

${ }^{3}$ Loc. cit. 
iodoform from diethylketone: $2 \mathrm{cc}$. of diethylketone are taken in a beaker and diluted with $150 \mathrm{cc}$. of weak ammonia. The mixture is warmed on the water bath and to it iodide solution is added when at first nitrogen iodide is precipitated, which is immediately taken up. The addition of iodine has to be continued, and finally a copious yellow crystalline precipitate deposits. This is iodoform. On recrystallization from alcohol, this has been obtained quite pure, melting at $119^{\circ}$. The yield amounts to $0.8 \mathrm{~g}$.

Acetylacetone.--Like the other ketones, it does not produce iodoform in concentrated solutions, but only a thick oil probably consisting of iodo derivative of the ketone. A dilute solution is used for the preparation of iodoform. $2 \mathrm{cc}$. of acetyl acetone are diluted with roo cc. of water and to it some ammonia is added. Then iodine solution is added to the hot solution. After a considerable quantity of iodine has been taken up, a yellow crystalline precipitate deposits at the bottom of the vessel, which consists of iodoform. The precipitate is filtered, washed, and recrystallized from alcohol, when it is obtained quite pure, melting at $119^{\circ}$.

Acetylmethylpropylketone.-When a fairly concentrated solution of the substance in excess of strong ammonia is acted upon by iodine solution, a brown oil is obtained, the examination of which is deferred for the present. For the preparation of iodoform, a dilute solution is used and the method of procedure is nearly the same as in the previous cases. At the end this is obtained as a yellow crystalline precipitate. On recrystallization from alcohol, it is obtained quite pure, melting at $119^{\circ}$, the yield obtained being good.

Acetylmethylhexylketone.-For the preparation of iodoform, the reaction is carried out as usual with a dilute solution of the ketone. At the conclusion of the process of iodination, a viscous mass is obtained from which the iodoform is secured by sucking off the viscous liquid. Crystallization from alcohol gives the pure product melting at $119^{\circ}$.

Acetoxime.-Acetoxime yields iodoform on treatment with nitrogen iodide. It has been found that during the interaction there was a copious evolution of nitrogen which is due to the decomposition of the $=\mathrm{NOH}$ group. It is quite likely that at first simple hydrolysis takes place producing hydroxylamine, which decomposes in the presence of iodine into nitrogen.

Acetophenoneoxime.-This yields iodoform and during the interaction a strong smell of benzaldehyde is noticed. The yield of iodoform is small.

Malonic Ester.-Malonic ester yields a good quantity of iodoform when treated with iodine and ammonia. To a dilute solution of the ester in ammonia, iodine solution is gradually added. On warming the 
iodine is taken up immediately and there is no intermediate precipitate of nitrogen iodide. After some time, a precipitate begins to appear which gradually increases as the process is continued. When the liquid is permanently brown, the operation is stopped. The precipitate consists of pure iodoform uncontaminated with any other secondary products. It melts at $119^{\circ}$.

Acetoacetic Ester.-On treating acetoacetic ester in strong aqueous solution, with iodine in the presence of ammonia, a viscous oil is obtained in good yield which contains no iodine and appears to be a condensation product of the ester. The substance is under investigation. As with other cases, iodoform is produced on carrying the interactions in dilute solutions. $2 \mathrm{cc}$. of acetoacetic ester are diluted with $\mathrm{I}_{5} \mathrm{O} \mathrm{cc}$. with some ammonia. Then the iodine solution is gradually added to the hot solution. In this case also, there is no immediate precipitation; the iodine is taken up as soon as it is added. At the end, a crystalline precipitate consisting of pure iodoform melting at $119^{\circ}$ is obtained.

Diethylamine.-Diethylamine yields iodoform on treatment with iodine and ammonia. To a dilute solution of the amine in ammonia iodine solution is gradually added when there appears an immediate precipitate of nitrogen iodide which is taken up on heating on the water bath and shaking. On continuing the operation until no more of iodine is taken up, yellow crystals of iodoform are obtained which on recrystallization from alcohol melt at II $9^{\circ}$.

Triethylamine.-Similarly to diethylamine, triethylamine has been found to yield iodoform in good yield. On addition of iodine solution, a brick red turbidity is produced which dissolves on shaking. A sticky liquid at first collects which goes on increasing as the action is continued. Finally the oil is gradually transformed into a yellow crystalline precipitate of iodoform. The precipitate is recrystallized from alcohol and is found to melt at $119^{\circ}$.

Ethyl and Propyl Alcohols.-Iodine in the presence of ammonia has no action on the alcohols, but the reagent acts in a complete manner on the sodium alcoholates.

Mesityl Oxide.-The formation of iodoform is successful in this case by taking a very dilute solution of mesityl oxide. In this case there is an intermediate precipitate of nitrogen iodide. At the end, a yellow crystalline precipitate consisting of iodoform is obtained which is obtained pure by recrystallization.

It appears from the very small number of cases which have been studied that the formation of iodoform is not limited in those compounds having a $\mathrm{CH}_{2} \mathrm{CO}$ - group as is generally supposed. The formation of iodoform appears to be a question of breaking up of molecules in iodinating decom- 
posing substances. More cases are being studied with a view to throw more light on the subject.

Investigations on similar lines are being continued.

Calcotta, India.

\title{
[Contribution from the Chemistry Department of Purdue University.]
}

\section{THE ETHYL-SULFURIC ACID REACTION.}

\author{
By P. N. Evans aNd J. M. AlBertson.
}

Received January 6, 1917.

Since the time of Williamson's classical work on etherification published in 1850 some of the reactions between ethyl alcohol and sulfuric acid have been well known. There are three more important reactions, leading, respectively, to ethyl-sulfuric acid, ethyl ether, and ethylene, as represented by the equations:

$$
\begin{gathered}
\mathrm{C}_{2} \mathrm{H}_{5} \mathrm{OH}+\mathrm{H}_{2} \mathrm{SO}_{4} \longrightarrow \mathrm{C}_{2} \mathrm{H}_{5} \mathrm{HSO}_{4}+\mathrm{H}_{2} \mathrm{O} \\
{ }_{2} \mathrm{C}_{2} \mathrm{H}_{5} \mathrm{OH} \longrightarrow \mathrm{C}_{2} \mathrm{H}_{5} \mathrm{OC}_{2} \mathrm{H}_{5}+\mathrm{H}_{2} \mathrm{O} \\
\mathrm{C}_{2} \mathrm{H}_{5} \mathrm{OH} \longrightarrow \mathrm{C}_{2} \mathrm{H}_{4}+\mathrm{H}_{2} \mathrm{O}
\end{gathered}
$$

Three other reactions of less importance are known, leading, respectively, to ethyl sulfate, isethionic acid, and ethionic acid, in accordance with the equations:

$$
\begin{gathered}
{ }_{2} \mathrm{C}_{2} \mathrm{H}_{5} \mathrm{OH}+\mathrm{H}_{2} \mathrm{SO}_{4} \longrightarrow\left(\mathrm{C}_{2} \mathrm{H}_{5}\right)_{2} \mathrm{SO}_{4}+\mathrm{H}_{2} \mathrm{O} \\
\mathrm{C}_{2} \mathrm{H}_{5} \mathrm{OH}+\mathrm{H}_{2} \mathrm{SO}_{4} \longrightarrow \mathrm{CH}_{2} \mathrm{OH} \cdot \mathrm{CH}_{2} \mathrm{SO}_{2} \mathrm{OH}+\mathrm{H}_{2} \mathrm{O} \\
\mathrm{C}_{2} \mathrm{H}_{5} \mathrm{OH}+{ }_{2} \mathrm{H}_{2} \mathrm{SO}_{4} \longrightarrow \mathrm{CH}_{2} \mathrm{HSO}_{4} \cdot \mathrm{CH}_{2} \mathrm{SO}_{2} \mathrm{OH}+{ }_{2} \mathrm{H}_{2} \mathrm{O}
\end{gathered}
$$

No attempt is here made to show the intermediate steps in the formation of the products mentioned; it is believed, however, that ethyl-sulfuric acid reacts with alcohol to form ether, as shown in the equation:

$$
\mathrm{C}_{2} \mathrm{H}_{5} \mathrm{HSO}_{4}+\mathrm{C}_{2} \mathrm{H}_{5} \mathrm{OH} \longrightarrow \mathrm{C}_{2} \mathrm{H}_{5} \mathrm{OC}_{2} \mathrm{H}_{5}+\mathrm{H}_{2} \mathrm{SO}_{4}
$$

The speed of these reactions varies with the temperature, that leading to ethyl-sulfuric acid (A), reaching equilibrium within two or three hours at $20^{\circ}$ and within ten minutes at $70^{\circ}$ or above, as will be shown later; the other reactions are very slow at ordinary temperatures; about $140^{\circ}$ is most favorable for the ether reaction (B), and about $160^{\circ}$ for the ethylene reaction $(\mathrm{C})$.

The completeness of the ethyl-sulfuric acid reaction (A) was shown by Claesson ${ }^{1}$ to be 57 to $59 \%$ with equimolecular quantities; the temperature is not stated in the abstracts accessible to the writer.

With a view to getting more detailed information as to the speed and degree of completeness of the ethyl-sulfuric acid reaction at different temperatures the work here reported was undertaken.

In this laboratory the formation of ether was examined as to its com-

1 J. prakt. Chem., [2] 19, 227 (1879). 